\title{
Development and characterization of an alginate-hyaluronic acid hydrogel for skin wound healing
}

Cristina Galocha-León ${ }^{1}$, Beatriz Clares-Naveros ${ }^{1,2}$, Noelia Perez-González ${ }^{1}$, Juan A. Marchal-Corrales ${ }^{2,3,4}$, Cristina Antich-Acedo ${ }^{2,3,4,5}$, Patricia Gálvez-Martín ${ }^{1,3,6}$,

${ }^{1}$ Department of Pharmacy and Pharmaceutical Technology, School of Pharmacy, University of Granada, Granada, Spain.

${ }^{2}$ Biosanitary Research Institute of Granada (ibs.GRANADA), University Hospitals of Granada-University of Granada, Granada, Spain.

${ }^{3}$ Biopathology and Regenerative Medicine Institute (IBIMER), Centre for Biomedical Research (CIBM), University of Granada, Granada, Spain.

${ }^{4}$ Department of Human Anatomy and Embryology, Faculty of Medicine, University of 6Granada, Granada, Spain.

${ }^{5}$ Excellence Research Unit "Modeling Nature" (MNat), University of Granada, Granada, Spain.

${ }^{6}$ Advance Therapies Area, Bioibérica S.A.U., Barcelona, Spain.

Background: The encapsulation of Mesemchymal Stem Cells (MSCs) into a hydrogel provides a promising future in diverse biomedical applications. MSCs provide a regenerative wound healing microenvironment due to their capacity to produce and secrete growth factors and cytokines that enhance granulation tissue formation, angiogenesis and reduce inflammation, which result in accelerated wound closure $^{1-2}$. Hydrogels are three-dimensional hydrophilic polymeric able to retain large amounts of water or biological fluids. Hydrogels possess similar structure to the extracellular matrix (ECM) and they are used in regenerative medicine due to their biocompatibility and their capability to act as a growth medium ${ }^{3}$. Hyaluronic acid (HA) is a non-sulfated glycosaminoglycan; it is also the main element of ECM. For this reason and have been investigated due to their biocompatibility, biodegradability and hydrophilic character. HA also lacks gelation abilities, thus it is used in combination with natural gelling agents such as alginate $(A)^{4}$.

Purpose: The aim of this work was the development and in vitro characterization of an alginate-hyaluronic acid based hydrogel for wound healing applications.

Methods: A-HA pre-gel solution was elaborated by dissolution of both autoclaved polymers in deionized water at concentrations of $2 \%$ and $1 \%(\mathrm{w} / \mathrm{v})$, respectively. Then, $1 \times 10^{6}$ cells $/ \mathrm{mL}$ was added on the pre-gel solutions and mixed until complete homogenization. Finally, $1 \%$ of $100 \mathrm{mM} \mathrm{CaCl}_{2}$ was added to obtain the hydrogel. Many important properties for the design of a hydrogel, such as swelling, degradation and porosity were studied. Swelling rate was assessed by a gravimetric method in phosphate buffer saline (PBS) at $\mathrm{pH} 7.4$ and $37{ }^{\circ} \mathrm{C}$. The degradation was calculated by incubating fresh hydrogels under the same conditions. The porosity analysis was carried out by immersion of the dry hydrogels in PBS pH 7.4 at room temperature.

Results and Discussion: The swelling ratio value was around $1500 \%$. The swelling behavior in wound healing can promote the transportation of nutrients and provide mechanical resilience to the delivery systems and the biological site of action. Degradation rate about $100 \%$ was reached in $300 \mathrm{~min}$. The degradation of the hydrogel should be progressive and takes place simultaneously with the restoration of the new tissue. The calculation of the porosity resulted in values of $52 \%$. It is an 
important characteristic of hydrogel because this highly porous structure allows and promotes nutrient transport, and facilitates cell proliferation and differentiation, which involves cell migrations.

Conclusions: These results confirm that this hydrogel present optimal characteristics to promote cell adhesion, proliferation and differentiation, thus it could be proposed as a suitable vehicle for cell delivery in tissue regeneration.

\section{References:}

${ }^{1}$ Murphy K.C., Whitehead J., Zhou D., Ho S.S., Leach J.K. (2017). Engineering fibrin hydrogels to promote the wound healing potential of mesenchymal stem cell spheroids. Acta Biomater, 64, 176-186.

${ }^{2} \mathrm{Hu}$ M.S., Borrelli M.R., Lorenz H.P., Longaker M.T., Wan D.C. (2018). Mesenchymal Stromal Cells and Cutaneous Wound Healing: A Comprehensive Review of the Background, Role, and Therapeutic Potential. Stem Cells International. Article ID 6901983.

${ }^{3}$ Tsou Y.H., Khoneisser J., Huang P.C., Xu X. (2016). Hydrogel as a bioactive material to regulate stem cell fate. Bioactive Materials, 1, 39-55.

${ }^{4}$ Antich, C., de Vicente, J., Jiménez, G., Chocarro, C., Carrillo, E., Montañez, E., Marchal, J. A. (2020). Bio-inspired hydrogel composed of hyaluronic acid and alginate as a potential bioink for 3D bioprinting of articular cartilage engineering constructs. Acta Biomaterialia, 106, 114-123. 